\title{
Probing topological order in quantum Hall states using entanglement calculations
}

\author{
Masudul Haque
}

April 2, 2008

\begin{abstract}
I provide the background and a brief review of the interdisciplinary use of entanglement calculations to investigate a type of unconventional order in many-body systems, known as topological order. The discussion focuses on a particular class of topologically ordered states: the fractional quantum Hall states
\end{abstract}

\section{Introduction}

Given the richness of 'emergent' phenomena in many-particle systems, it is perhaps no surprise that some quantum many-particle states continue to resist the methods of analysis that condensed matter theory has developed till now. Important examples are topologically ordered states [2] and non-Fermi liquid states. New methods and tools are therefore essential in understanding such states and phenomena. In an unexpected twist, concepts from a completely different and much younger field, quantum information theory, are proving to be useful in this regard. In this brief review we will focus on the use of entanglement measures from quantum information to study topological order. An entanglement measure known as the entanglement entropy, when calculated for judiciously chosen bipartitions of the topologically ordered state, provides a physically significant topological quantum number associated with the state. Currently, topological order is receiving intense attention due to quantum computation proposals based on the phenomenon [1]; a novel probe for such order is an important and timely development.

We will describe entanglement calculations in the most prominent type of topologically ordered state, namely, fractional quantum Hall states.

\section{Topological order and quantum Hall states}

Over half a century, condensed matter theory has developed a mature, widely used, paradigm for understanding many-particle phases and phase transitions between them - using symmetries to distinguish between phases and correlation functions 
to characterize them. However, some states of matter continue to defy the conventional description. One important example is topologically ordered states [2], which are notoriously difficult to describe within the standard 'Landau' paradigm.

Topologically ordered states are 'gapped' in their low-energy excitation spectrum, and are further characterized by ground-state degeneracy on higher-genus surfaces, and in some cases by fractionalized excitations. For example, on a genus- $g$ surface, the Laughlin state at filling $\nu=1 / m$ has $m^{g}$ ground states. The topologydependent degeneracy is intimately connected to fractionalization [3].

The two physical contexts for topological order are frustrated magnet systems and quantum Hall systems. In the magnetic context, topological order remains a theoretical prospect, as there are no experimental realizations. A number of topologically ordered magnetic states have been constructed theoretically; e.g., (1) Kitaev's models [4]; (2) quantum dimer models [5] on non-bipartite lattices [6]; (3) the chiral spin liquid of Laughlin and Kalmeyer [7]; (4) spin liquid states obtained by Gutzwiller-projecting BCS states [8].

The only confirmed experimental realizations of topological order are the fractional quantum Hall (FQH) states occurring in two-dimensional (2D) electrons in a magnetic field $[9,2]$. Realizations of FQH states have also been proposed in cold-atom setups [10]. FQH states have long fascinated the condensed-matter community due to their remarkable transport properties and the exotic nature of their quasiparticle excitations. Recently there has been enhanced interest in FQH states with non-abelian statistics $[11,12,13]$, due to the possibility of implementing quantum computation schemes topologically protected from decoherence [1]. The unusual features of FQH states have been notoriously difficult to characterize using traditional condensed-matter concepts such as local order parameters and $n$-point correlation functions. In this review we will focus on an alternate characterization of FQH states, via a recent connection found between topological order and entanglement entropy.

\section{Entanglement entropy in condensed matter}

The entanglement entropy is one of several possible measures of bi-partite quantum entanglement between partitions $A$ and $B$ of a quantum state. It is defined as $S_{A}=-\operatorname{tr}\left[\rho_{A} \ln \rho_{A}\right]$, in terms of the reduced density matrix $\rho_{A}=\operatorname{tr}_{B} \rho$ obtained by tracing out $B$ degrees of freedom from the system density matrix $\rho$. If $A$ and $B$ are two spins (qubits), one finds $S_{A}=0$ for product states like $|\uparrow \uparrow\rangle,|\uparrow \downarrow\rangle$, and in contrast $S_{A} \neq 0$ for entangled states such as $(|\uparrow \downarrow\rangle \pm|\downarrow \uparrow\rangle)$ or $\left(c_{1}|\uparrow \uparrow\rangle+c_{2}|\downarrow \downarrow\rangle\right)$.

In the original (quantum information) setting, partitioning is often obvious: $A$ and $B$ can be just two qubits or two qutrits. For a many-particle system, there are exponentially many ways of partitioning. A challenge is to decide which partitioning will lead to physically interesting information. One prominent example is the study of the entanglement between a block $(A)$ and the rest $(B)$ of a manyparticle system, measured by the entanglement entropy $S_{A}$, as a function of the block size (Fig. 1). For such block partitioning of many-particle ground states, the general rule ('area law') is that the entanglement entropy scales as the size of the 

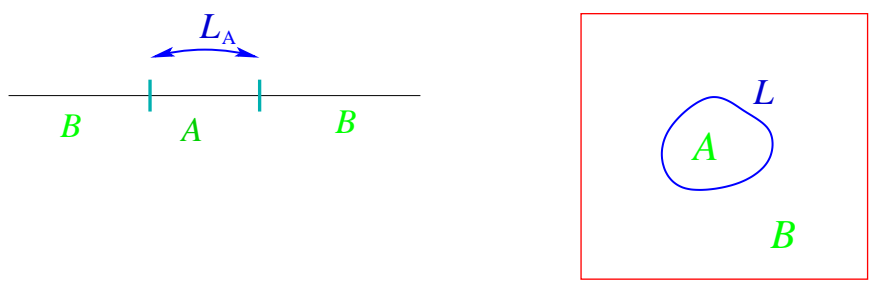

Figure 1: Partitioning of many-particle systems into blocks $A$ and $B$, between which the entanglement $\left(S_{A}\right)$ is calculated. Entanglement $S_{A}$ is studied as function of boundary length $L$, in the asymptotic limit $L \rightarrow \infty$. Left: Block partitioning in 1D. Asymptotic behavior distinguishes between gapless and gapped states. For critical states, asymptotics gives central charge of conformal field theory [15]. Right: Block partitioning in 2D, relevant to the topologically ordered states.

boundary between the $A$ and $B$ blocks $[14,15]$. Subtle information about the nature of the many-particle state can be contained in the coefficients, logarithmic corrections, or subleading terms in this basic relationship. For one-dimensional (1D) systems, the asymptotic behavior of $S_{A}$ (Fig. 1 left) provides a distinction between gapless and gapped states, a result widely known and well-exploited by now [15]. More recently, and more pertinent to this review, the block entanglement entropy is also showing promise for exploring unconventional states in two dimensions (Fig. 1 right), such as topologically ordered states [16, 17].

\section{The topological entanglement entropy}

For topologically ordered states in two dimensions, the following theorem has been presented recently $[16,17]$. If $L$ is the length of the boundary between the two blocks (e.g., Fig. 1 right), the entanglement entropy in the large- $L$ limit scales as

$$
S_{A}=\alpha L-\gamma+\mathcal{O}\left(L^{-1}\right) \text {. }
$$

As usual the scaling relationship applies to situations where $A$ is large and the total system is infinite. The subleading term $\gamma$ is called the topological entanglement entropy. This quantity is the logarithm of the so-called total quantum dimension of the topological field theory describing the topological order of the state. The total quantum dimension $\mathcal{D}$ is given by $\mathcal{D}=\left(\sum_{i} d_{i}^{2}\right)^{1 / 2}$, where the $d_{i}$ 's are the quantum dimensions of the individual sectors making up the topological field theory. These individual quantum dimensions are set by fusion rules of the fundamental anyons in the field theory, as illustrated in Sec. 5 by examples in the FQH context.

After the concept was introduced [16, 17], the topological entanglement entropy has been calculated for several topologically ordered systems, notably quantum dimer models [18], Kitaev models [19, 20], and FQH systems [21, 22, 23]. 


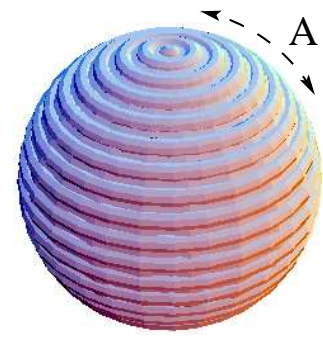

Figure 2: Spherical geometry for finite-size FQH calculations; partitioning into regions $A$ and rest $(B)$.

\section{Topological entanglement entropy for fractional quantum Hall systems}

The topological field theory for a $\nu=1 / m$ Laughlin state has a fundamental anyon (of fractional charge $1 / m$ ), which generates $m$ abelian sectors. The quantum dimension is unity in all sectors $\left(d_{i}=1\right)$. Thus for the $\nu=1 / m$ abelian Laughlin state, the total quantum dimension is $\mathcal{D}=\sqrt{m}$ and the topological entanglement entropy is $\gamma=\ln \sqrt{m}$. For $m=3$ this gives $\gamma=\frac{1}{2} \ln 3 \simeq 0.55$.

The situation is more interesting for states with non-abelian quasiparticles, because some anyon sectors now contribute $d_{i}>1$. Some examples have been detailed in Refs. [16, 24, 22]. In particular, for the $m=2$ Moore-Read state, there are six sectors, two each of quasiparticles denoted by $I, \sigma, \psi$. These contribute $d_{I}=1, d_{\sigma}=\sqrt{2}, d_{\psi}=1$, leading to $\mathcal{D}=\sqrt{8}$ and $\gamma=\ln \sqrt{8} \simeq 1.04$. The nonabelian nature shows up in the fact that $\gamma$ is larger than $\ln \sqrt{6}$, which would be expected if there were six merely abelian sectors.

\subsection{Explicit calculations}

Extracting the topological entanglement entropy $\gamma$ explicitly from quantum Hall wavefunctions is a complicated problem. Numerically, this has been done in Refs. [21, 22] for explicitly constructed Laughlin [9] and Moore-Read [11] states, and in Ref. [23] for the ground states of Coulomb Hamiltonians at appropriate filling.

When working with finite-size FQH wavefunctions, one has to make a choice of geometry, generally avoiding systems with boundaries. (FQH states have nontrivial edge effects.) The common choices are spherical and toroidal geometries, used respectively in Refs. [21, 22] and in Ref. [23]. In each case, the finite-size numerical data has to be extrapolated to the thermodynamic limit. The spherical geometry (used in Refs. [21, 22]) involves magnetic orbitals shaped as "lines of latitude" [25, 26]. Fig. 2 shows the choice of partition $A$ used in Refs. [21, 22]. Using entanglement data for this type of partitioning in finite-size FQH wavefunctions, $\gamma$ can be extracted after extrapolation to the macroscopic limit. Extrapolation remains a tricky issue and improvements are being developed compared to the methods used in Refs. [21, 22]. 

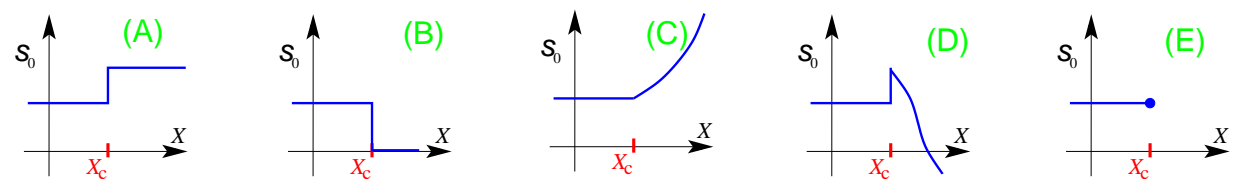

Figure 3: Intercept of block entropy $S_{A}(L)$ at phase transition. The $X<X_{\mathrm{c}}$ region is a topologically ordered phase.

\section{$5.2 \quad$ Prospective uses}

The successful explicit calculation of the topological entanglement entropy $\gamma$ for model states and Coulomb ground states [21, 22, 23] opens up the exciting possibility of using $\gamma$ as a novel tool to study quantum Hall physics. Prospective uses are outlined below.

First, one can hope to use entanglement calculations to probe quantum phase transitions between FQH and non-FQH ground states. For the block entanglement entropy, let us imagine that we have determined the asymptotic relationship

$$
S_{(L \rightarrow \infty)} \quad \longrightarrow \alpha L-s_{0}
$$

where $L$ is the boundary of the block. Note that this is not necessarily always possible; in some $2 \mathrm{D}$ phases the leading term might not be purely linear.

In a topologically ordered phase, the negative intercept $s_{0}$ will by definition be equal to the topological entropy, $\gamma=\ln \mathcal{D}$. Fig. 3 shows what can happen to $s_{0}$ as the 2D system is driven across a quantum phase transition away from the topologically ordered state, by varying a parameter $X$ across the critical value $X_{\mathrm{c}}$. In the parameter range $X<X_{\mathrm{c}}$ where the system is in the topologically ordered phase, $s_{0}$ is fixed at a positive plateau $\left(s_{0}=\gamma\right)$.

Case A shows a transition into another topologically ordered state with a different quantum dimension; $s_{0}$ jumps to another constant value $\gamma^{\prime}$. The other figures show transitions to non-topological phases. Case B shows a transition to a gapped state which is not topologically ordered - the intercept drops to zero. Cases $\mathrm{C}$ and $\mathrm{D}$ show continuous and discontinuous transitions into non-topological phases, in which the negative intercept is nonzero but not constant. Finally, Case E shows a transition into a state where the leading term in the asymptotic behavior of $S_{A}(L)$ is not linear, so that $s_{0}$ as is undefined.

A second exciting prospect is to use $\gamma$ calculations to test which conformal field theories are appropriate for certain states. There are FQH states (e.g., composite fermion states) for which the underlying field theory is not clear. Since $\gamma$ is related to the quantum dimensions $\left(d_{i}\right)$ of the theory, such determination of $\gamma$ would place strong bounds on acceptable conformal field theories.

Finally, for quantum Hall fractions at which there are more than one candidate theoretical state, $\gamma$ calculations could be used to distinguish between proposed candidate states if they have different total quantum dimension. 


\section{References}

[1] M. Freedman, M. Larsen, and Z. Wang; Commun. Math. Phys. 227, 605 (2002). L. B. Ioffe et. al.; Nature 415, 503 (2002). S. Das Sarma, M. Freedman, and C. Nayak; Phys. Rev. Lett. 94, 166802 (2005); also Physics Today, July 2006, p. 32. A. Yu. Kitaev, Ann. Phys. 303, 2 (2003). S. Das Sarma et. al., arXiv:0707.1889.

[2] X. G. Wen \& Q. Niu; Phys. Rev. B 41, 9377 (1990).

X. G. Wen, Phys. Rev. B 40, 7387 (1989); J. Math. Phys. 4, 239 (1990).

X. G. Wen; Quantum Field Theory of Many-body Systems, Oxford, 2004.

[3] Oshikawa \& Senthil; Phys. Rev. Lett. 96, 060601 (2006).

[4] A. Yu. Kitaev; Ann. Phys. 303, 2 (2003); Ann. Phys. 321, 2 (2006).

[5] S. A. Kivelson, D. S. Rokhsar, and J. P. Sethna, Phys. Rev. B 35, 8865 (1987). D. S. Rokhsar and S. A. Kivelson, Phys. Rev. Lett. 61, 2376 (1998).

[6] D. S. Rokhsar and S. A. Kivelson, Phys. Rev. Lett. 61, 2376 (1998). R. Moessner and S. L. Sondhi, Phys. Rev. Lett. 86, 1881 (2001). G. Misguich, D. Serban, and V. Pasquier, Phys. Rev. Lett. 89, 137202 (2002).

[7] V. Kalmeyer and R. B. Laughlin; Phys. Rev. Lett. 59, 2095 (1987); Phys. Rev. B 39, 11879 (1989). R. B. Laughlin, Ann. Phys. (N.Y.) 191, 163 (1989). R. B. Laughlin and Z. Zou, Phys. Rev. B 41, 664 (1990).

[8] D. A. Ivanov and T. Senthil, Phys. Rev. B 66, 115111 (2002).

Paramekanti et. al., Phys. Rev. B 71, 094421 (2005).

[9] D. C. Tsui, H. L. Stormer, and A. C. Gossard; Phys. Rev. Lett. 48, 1559 (1982). R.B . Laughlin; ibid. 50, 1395 (1983).

[10] N. K. Wilkin, J. M. F. Gunn, and R. A. Smith, Phys. Rev. Lett. 80, 2265 (1998). N. R. Cooper and N. K. Wilkin, Phys. Rev. B 60, R16279 (1999). N. Regnault and Th. Jolicoeur, Phys. Rev. B 70241307 (2004). A. S. Sorenson, E. Demler, and M. D. Lukin; Phys. Rev. Lett. 94, 086803 (2005).

[11] G. Moore and N. Read, Nucl. Phys. B360, 362 (1991).

[12] N. Read and E. Rezayi, Phys. Rev. B 59, 8084 (1999).

[13] E. Ardonne and K. Schoutens, Phys. Rev. Lett. 82, 5096 (1999).

[14] M. Srednicki, Phys. Rev. Lett. 71, 666 (1993).

[15] G. Vidal, J. I. Latorre, E. Rico and A. Kitaev, Phys. Rev. Lett. 90, 2279021 (2003). V.E. Korepin; Phys. Rev. Lett. 92, 096402 (2004). P. Calabrese and J. Cardy; J. Stat. Mech. 0406, 002 (2004).

[16] A. Kitaev \& J. Preskill; Phys. Rev. Lett. 96, 110404 (2006). 
[17] M. Levin \& X. G. Wen; ibid. 96, 110405 (2006).

[18] S. Furukawa \& G. Misguich; Phys. Rev. B 75, 214407 (2007).

[19] C. Castelnovo and C. Chamon, Phys. Rev. B 76, 184442 (2007).

[20] C. Castelnovo and C. Chamon, Phys. Rev. B 77, 054433 (2008).

[21] M. Haque, O. Zozulya and K. Schoutens; Phys. Rev. Lett. 98, 060401 (2007).

[22] O. S. Zozulya, M. Haque, K. Schoutens, and E. H. Rezayi; Phys. Rev. B 76, 125310 (2007).

[23] B.A. Friedman and G. C. Levine, eprint arXiv:0710.4071.

[24] P. Fendley, M. P. A. Fisher, and C. Nayak, J. Stat. Phys. 126, 1111 (2007).

[25] F. D. M. Haldane, Phys. Rev. Lett. 51, 605 (1983).

[26] D. P. Arovas, A. Auerbach, and F. D. M. Haldane, Phys. Rev. Lett. 60, 531 (1988). 